\title{
Association between high mobility group box-1 protein expression and cell death in acute pancreatitis
}

\author{
ENJUN GAO, YANFENG JIANG, ZHITUO LI, DONGBO XUE and WEIHUI ZHANG \\ Department of General Surgery, The First Affiliated Hospital of Harbin Medical \\ University, Harbin, Heilongjiang 150001, P.R. China
}

Received February 8, 2016; Accepted February 20, 2017

DOI: $10.3892 / \mathrm{mmr} .2017 .6496$

\begin{abstract}
The present study used caerulein stimulation of AR42J rat pancreatic cells as an in vitro acute pancreatitis (AP) model to investigate proteins differentially expressed in apoptosis and necrosis. AR42J cells were stimulated with $10^{-8} \mathrm{~mol} / \mathrm{l}$ caerulein and incubated for $24 \mathrm{~h}$. Apoptosis and necrosis were detected using flow cytometry. The sorted Annexin V-positive cells (apoptotic) and the Annexin V/propidium iodide double-positive cells (necrotic) were analysed using proteomics. Results showed that numerous proteins were differentially expressed in these 2 groups. Functional enrichment analysis was performed on the differentially expressed genes using the Database for Annotation, Visualization and Integrated Discovery. High mobility group box-1 protein (HMGB1) was specifically expressed in the necrosis group. Models of varying degrees of AP were established using caerulein at concentrations of $10^{-9}, 10^{-8}, 10^{-7}, 10^{-6}$ and $10^{-5} \mathrm{~mol} / \mathrm{l}$. The percentage of apoptotic and necrotic cells in each group was determined using flow cytometry. Protein expression levels of HMGB1 were detected by western blot analysis. The present study showed that as the concentration of caerulein increased, the percentage of necrotic cells and the protein expression levels of HMGB1 increased. HMGB1 is involved in many biological processes, including the chromosomal protein glycyl lysine isopeptide cross-link. HMGB1 may be involved in the early stage of pancreatitis, potentially by inducing the development of cell death by necrosis. These results provide an experimental basis for clinical intervention in AP.
\end{abstract}

\section{Introduction}

Acute pancreatitis (AP) is an acute inflammation of the pancreas. The primary clinical presentation is acute abdominal

Correspondence to: Professor Weihui Zhang or Professor Dongbo Xue, Department of General Surgery, The First Affiliated Hospital of Harbin Medical University, 23 Youzheng Street, Harbin, Heilongjiang 150001, P.R. China

E-mail: zhangweihui626@hotmail.com

E-mail: dongbo_xue@126.com

Key words: acute pancreatitis, caerulein, apoptosis, necrosis, proteomics, high mobility group box-1 protein pain combined with an increase in serum amylase. For $\sim 80 \%$ of patients, the disease is self-limiting, whereas the remainder may develop severe acute pancreatitis (SAP), the mortality rate of which is $\sim 30 \%$. The pathogenic underlying mechanisms remain to be fully elucidated. Previous theories of trypsin autodigestion (1), pancreatic microcirculation dysfunction (2), free radical damage (3) and cell apoptosis (4) do not fully account for the disease. Rinderknecht (5) proposed the 'inflammatory mediator' theory. According to this theory, a large number of cytokines released by cells induces a complex inflammatory cascade, which mediates damage in the pancreas and in extrapancreatic organs, thus causing a systemic inflammatory reaction.

Trypsin autodigestion is considered to be the primary process involved in AP. Even in the early stages of AP, pancreatic acinar cell damage occurs, and the type of acinar cell death is important for the development and prognosis of AP. Cell death type may influence the release of trypsin from acinar cells, thus indirectly affecting the degree of pancreatitis. Therefore, control of the activation and release of trypsin has become critical for treating pancreatitis. The types of pancreatic cell death include apoptosis and necrosis. If cells die from apoptosis, the cell membrane remains intact; therefore, there is no accompanying release of inflammatory factors and trypsin, and the inflammatory reaction is milder. By contrast, necrosis is accompanied by the release of intracellular contents and lysosomal enzymes. Particularly for pancreatic acinar cells, necrosis is associated with the release and activation of trypsin (6), which may induce the aggregation of a variety of inflammatory cells and cause severe inflammatory reactions (7).

The function of apoptosis and necrosis in AP is complex. An understanding of the factors involved in these two types of cell death may indicate a strategy for the promotion of acinar cell death by apoptosis, rather than necrosis, which may be important for the treatment of AP. The present study aimed to investigate proteins differentially expressed in apoptosis and necrosis in the AR42J rat pancreatic cell line.

\section{Materials and methods}

Establishment of the AP cell model. The AR42J rat pancreatic acinar cell line was purchased from the China Center for Type Culture Collection (Wuhan, China). Cells were cultured 
in Ham's F12 K (Kaighn's) culture medium (Gibco; Thermo Fisher Scientific, Inc., Waltham, MA, USA) supplemented with $10 \%$ foetal bovine serum (HyClone; GE Healthcare Life Sciences, Logan, UT, USA) in a $5 \% \mathrm{CO}_{2}$ incubator at $37^{\circ} \mathrm{C}$. Approximately $1 \times 10^{5} / \mathrm{ml}$ ARJ42 cells were seeded into 6 -well plates and cultured for $24 \mathrm{~h}$. Caerulein $\left(10^{-8} \mathrm{~mol} / \mathrm{l}\right.$, Sigma-Aldrich; Merck KGaA, Darmstadt, Germany) was added, and the cells were incubated for a further $24 \mathrm{~h}$. Finally, the ARJ42 cells were collected, and the percentages of apoptosis and necrosis were detected using a flow cytometer.

Labelling and flow cytometry sorting of apoptotic and necrotic cells. For fluorescence staining, ARJ42 cells were detached and collected in EDTA-free trypsin, washed twice with PBS and centrifuged at $106 \mathrm{x}$ g for $5 \mathrm{~min}$ at room temperature. A total of $5 \times 10^{5}$ cells were resuspended in $500 \mu \mathrm{l}$ binding buffer (Beijing Biosea Biotechnology Co., Ltd., Beijing, China). Following this, $5 \mu \mathrm{l}$ Annexin V-fluorescein isothiocyanate (FITC) and $5 \mu \mathrm{l}$ propidium iodide (PI) (Beijing Biosea Biotechnology Co., Ltd.) were added and mixed sequentially, and cells were incubated at room temperature in the dark for $15 \mathrm{~min}$.

For liquid flow monitoring, a 70-mm nozzle was selected, and the amplitude was adjusted to make the liquid flow break point between $1 / 2$ and $1 / 3$ of the window to ensure the regular shape of all of the primary liquid drops. The vibration amplitude of the liquid drops was adjusted to allow the Gap value to be as stable as possible. The satellite points were $<5$ and were fused into the liquid drops. The Amp1 value was 9.1, the Drop1 value was 252, and the Gap value was 7 .

For adjustment of the sorting liquid path, the voltage of the side liquid flow window was opened, and the Test Sort test was activated. The amplitude of the primary liquid flow window was gradually adjusted to clear the liquid flow separation. The adjusted Drop1 value in the primary liquid flow window was added to the default window. The Gap1 value, following liquid flow adjustment, should be within the range of the default value \pm 3 . The Amp1 value was 9.1, the 2 nd value was 14 , and the 3 rd value was 7 .

For confirmation of liquid drop delay, the original adjustment in precision was selected. The brightness in the left frame was adjusted to $100 \%$. The fine adjustment in precision was selected, and the liquid drop delay value was adjusted to make the brightness in the left frame $\geq 90 \%$. Finally, during the adjustment of the Drop Delay, following each single click, the adjustment was conducted after the system reacted to the liquid drop delay time. The Drop Delay value was 45.18.

Proteomics detection of apoptotic cells and necrotic cells. Sorted Annexin V-positive cells (V group) or the Annexin V/PI double-positive cells (VP group) at a density of $5 \times 10^{5}$ were mixed with $500 \mu \mathrm{l}$ 2D lysis buffer (Beyotime Institute of Biotechnology, Shanghai, China). Cells were sonicated (80 W, 5-sec pulses; repeated three times). Protein concentrations were determined using the Bradford method. Protein lysates were aliquoted into tubes (100 $\mu \mathrm{g} /$ tube). Samples $(20 \mu \mathrm{l})$ were loaded into each well and separated by electrophoresis at $200 \mathrm{~V}$ for $45 \mathrm{~min}-1 \mathrm{~h}$ on a $12 \%$ SDS-PAGE gel. Gel samples were stained with silver nitrate for $1-2 \mathrm{~h}$ and destained. The proteins in the gels were enzymatically digested; gel samples were mixed with $5 \mu \mathrm{l}$ Trypsin (10 ng/ $\mu \mathrm{l}$, Gibco; Thermo Fisher
Scientific, Inc.), buffer was added at $37^{\circ} \mathrm{C}$ overnight and the protein solution was aspirated into EP tube for the next step.

The $\operatorname{Ettan}^{\text {Tix }}$ Multidimensional Liquid Chromatography system (GE Healthcare Bio-Sciences, Pittsburgh, PA, USA) was used for desalting and separation of tryptic peptides mixtures. In this system, samples were desalted on Reversed-Phase (RP) Trap Columns (Zorbax 300SB-C18; Agilent Technologies, Inc., Santa Clara, CA, USA), and subsequently separated on an RP column (RP-C18; $150 \mu \mathrm{m} \times 100 \mathrm{~mm}$; Column Technologies, Lombard, IL, USA). Mobile phases A ( $0.1 \%$ formic acid in high performance liquid chromatography-grade water) and $\mathrm{B}(0.1 \%$ formic acid in acetonitrile) were selected. Tryptic peptide mixtures $(20 \mu \mathrm{g})$ were loaded onto the columns and separation was performed at a flow rate of $2 \mu \mathrm{l} / \mathrm{min}$ using a linear gradient of $4-50 \%$ B for 120 min. A Finnigan ${ }^{\text {TN }}$ LTQ-VELOS $^{\text {Tx }}$ Linear Ion Trap mass spectrometer (Thermo Electron Corporation, Beverly, MA, USA) equipped with an electrospray interface was connected to the liquid chromatography setup for eluted peptide detection. Data-dependent mass spectrometry (MS)/MS spectra were obtained simultaneously. Each scan cycle consisted of one full MS scan in profile mode followed by ten MS/MS scans in centroid mode with the following Dynamic Exclusion $^{\text {TI }}$ (Thermo Electron Corporation) settings: Repeat count, 2; repeat duration, $30 \mathrm{sec}$; and exclusion duration, 90 sec.

Bioinformatics analysis. The Database for Annotation, Visualization and Integrated Discovery (DAVID; https://david. abcc.ncifcrf.gov/) was used to screen differentially expressed genes for Gene Ontology-Biological Process (GO-BP) and pathway enrichment analysis based on the Kyoto Encyclopedia of Genes and Genomes (KEGG) database. The 'Functional Annotation Tool' was used for analysis. Differentially expressed genes previously identified were used for the GO-BP term and pathway analysis. The results were arranged according to the negative logarithms of the P-values. Cytoscape software version 2.6.3 (www.cytoscape.org/download.php) was used to plot the network graphs of corresponding genes and the GO-BP term and KEGG pathway.

The gene list of differentially expressed proteins was uploaded onto DAVID. In addition, the corresponding gene identifier was selected, and the rat whole genome was selected for the background genes. The 'Functional Annotation Tool' was selected as the analysis tool. The results of the functional enrichment analysis were then obtained. $\mathrm{P}<0.05$ was considered to indicate a statistically significant difference.

Detection of type of cell death of AR2J cells using Annexin VIPI double staining. AR42J cells $\left(5 \times 10^{6}\right.$ cell $\left./ \mathrm{ml}\right)$ were seeded into culture plates and incubated for $24 \mathrm{~h}$, in a $5 \% \mathrm{CO}_{2}$ incubator at $37^{\circ} \mathrm{C}$. Caerulein $\left(10^{-9}, 10^{-8}, 10^{-7}, 10^{-6}\right.$ or $\left.10^{-5} \mathrm{~mol} / \mathrm{l}\right)$ was added to the AR42J cells. The control group did not receive caerulein. According to the experimental results of our related research (8), the number of apoptotic cells decreased after the $8 \mathrm{~h}$ timepoint, so this was the timepoint selected. All 6 groups of cells were incubated for $8 \mathrm{~h}$. Following this the cells were collected and apoptosis and necrosis were detected using a flow cytometer.

Fluorescence staining was performed as aforementioned. For flow cytometry, cells were evaluated at an excitation 


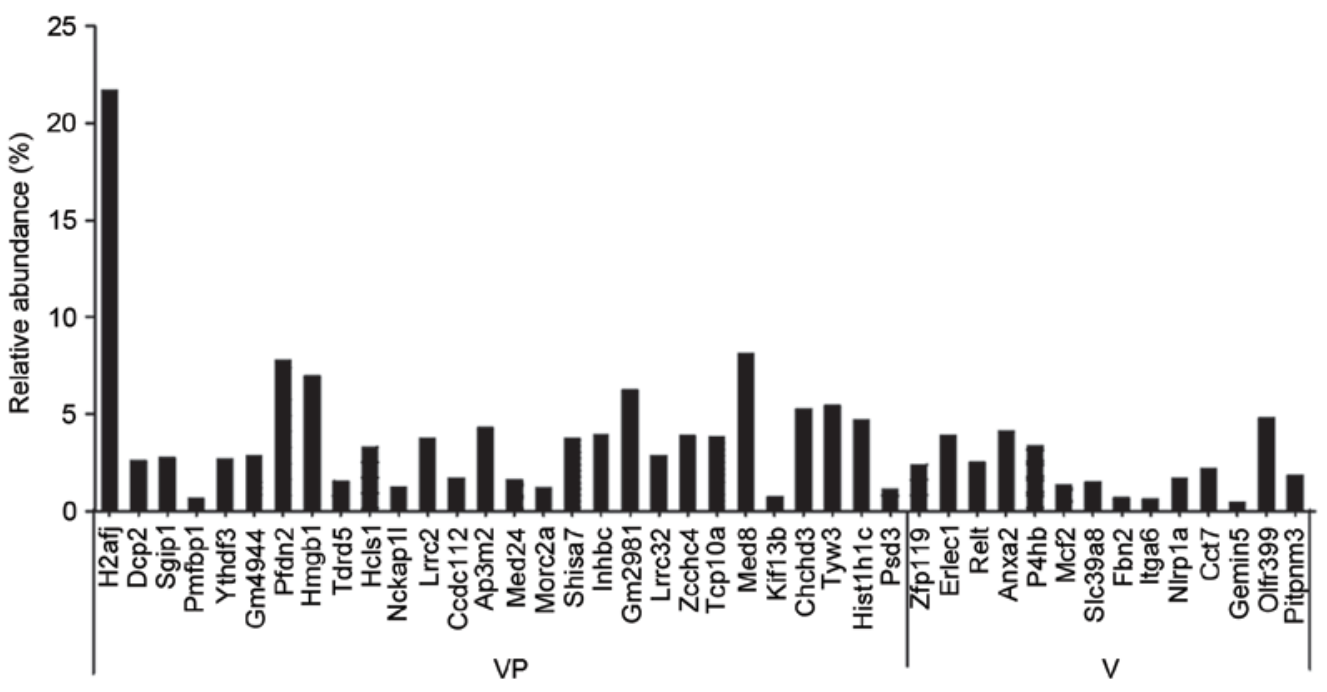

Figure 1. Proteomics analysis of apoptotic and necrotic pancreatic cells. The relative abundance of proteins differentially expressed in apoptotic and necrotic cells. V, apoptosis group; VP, necrosis group.

wavelength of $488 \mathrm{~nm}$, and an emission wavelength of $530 \mathrm{~nm}$. The green fluorescence of Annexin V-FITC was detected using the FITC channel (FL1), and the red fluorescence of PI was detected using the PI channel (FL2). WinMDI version 2.8 (http://www.cyto.purdue.edu/flowcyt/software/Winmdi.htm) was used for flow cytometric analysis.

Detection of high mobility group box-1 protein (HMGB1) expression. To validate the accuracy of the biological analyses, HMBG1 protein expression levels were detected by western blot analysis. Cells were treated with various concentrations of caerulein as aforementioned. Following treatment, cells were lysed with radioimmunoprecipitation assay buffer $[0.15 \mathrm{M} \mathrm{NaCl}$ and $0.05 \mathrm{mM}$ Tris- $\mathrm{HCl}(\mathrm{pH} 7.2)$ containing $1 \%$ NP-40, $1 \%$ sodium deoxycholate and $0.1 \%$ sodium dodecyl sulfate (SDS)] with $1 \mathrm{mM}$ phenylmethylsulfonyl fluoride, and subsequently centrifuged at $10,000 \mathrm{x} \mathrm{g}$ at $4^{\circ} \mathrm{C}$ for $10 \mathrm{~min}$. Protein concentrations were quantified using a Bicinchoninic Acid Protein assay kit (Beyotime Institute of Biotechnology, Haimen, China). Proteins (40-50 $\mu \mathrm{g}$ ) were separated by $12 \%$ SDS-PAGE gel and subsequently transferred onto polyvinylidene difluoride membranes. The membranes were incubated for $1 \mathrm{~h}$ at room temperature with a protein blocker (5\% skimmed milk in TBS containing Tween 20) to occupy the spaces on the membranes that were not occupied by protein. Then the membranes were incubated with the following primary antibodies at $4^{\circ} \mathrm{C}$ overnight: Rabbit anti-HMBG1 (3935s; 1:200; Cell Signaling Technology, Inc., Danvers, MA, USA) and rabbit anti- $\beta$-actin (WL0002; 1:200; Wanleibio, Co., Ltd., Shanghai, China). Following this, membranes were incubated with a goat anti-rabbit horseradish peroxidase-conjugated IgG secondary antibody (WLA023; 1:1,000; Wanleibio, Co., Ltd.). Proteins were subsequently detected with an Enhanced Chemiluminescence Plus kit (Beyotime Institute of Biotechnology) and the optical density values of target bands were assessed using Gel-Pro Analyzer software (version 6.3; Media Cybernetics, Inc., Rockville, MD, USA).

\section{Results}

Proteomics analysis of apoptotic and necrotic pancreatic cells. Annexin V-positive cells (V group) and Annexin V/PI double-positive cells (VP group) were sorted by flow cytometry and subjected to proteomics analysis. The results demonstrated that there were 28 different proteins specifically expressed in necrotic cells (VP group), including $\mathrm{H} 2 \mathrm{~A}$ histone family member J (H2AFJ), mRNA-decapping enzyme 2 (DCP2), SH3-domain GRB2-like (endophilin) interacting protein 1 (SGIP1), polyamine modulated factor 1 binding protein 1 (PMFBP1), YTHDF3, zinc finger protein 994 (GM4944), prefoldin subunit 2 (PFDN2), HMGB1, tudor domain containing 5 (TDRD5) and hematopoietic linage cell-specific protein (HCLS1). There were 14 different proteins specifically expressed in apoptotic cells (V group), including endoplasmic reticulum lectin 1 (ERLEC1), tumour necrosis factor receptor superfamily member 19L (RELT), Annexin A2 (ANXA2), protein disulphide-isomerase (P4HB), proto-oncogene MCF-2 (MCF2) and solute carrier family 39 member 8 (SLC39A8; Fig. 1).

Functional analysis of genes associated with differentially expressed proteins. The results revealed that HMGB1 was associated with numerous biological processes, including the formation of the chromosomal protein glycyl lysine isopeptide cross-link, and the positive regulation of phosphorylation, protein acid phosphorylation, the phosphate metabolic process and the phosphorus metabolic process (Fig. 2).

Flow cytometric analysis of AR42J cells stimulated with various concentrations of caerulein. AR42J cells treated with 0 (Fig. 3A), $10^{-9}$ (Fig. 3B), $10^{-8}$ (Fig. 3C), $10^{-7}$ (Fig. 3D), $10^{-6}$ (Fig. 3F) or $10^{-5} \mathrm{~mol} / \mathrm{l}$ (Fig. 3F) caerulein were assessed for apoptosis and necrosis. As the concentration of caerulein increased from $10^{-9}$ to $10^{-5} \mathrm{~mol} / 1$, the percentage of necrotic cells increased, and cell necrosis frequency increased from 1.3 to $15.04 \%$ (Fig. 3G). No obvious change was observed in the 


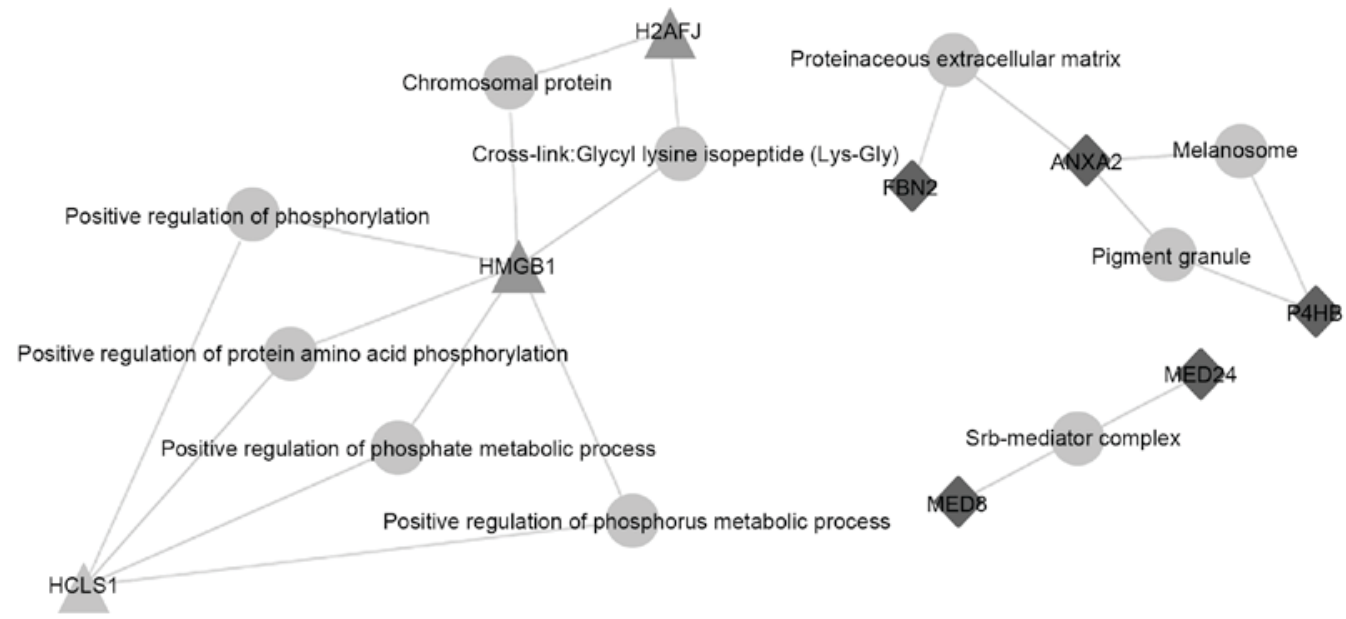

Figure 2. Functional analysis of genes encoding differentially expressed proteins. Schematic of genes and associated signal transduction networks in apoptotic and necrotic cells: grey circles, biological processes; grey triangles, proteins specifically expressed in necrotic cells; black diamonds, proteins specifically expressed in apoptotic cells. H2AFJ, H2A histone family member J; HMGB1, high mobility group box-1 protein; HCLS1, hematopoietic linage cell-specific protein 1. FBN2, fibrillin 2; ANXA2, annexin A2; P4HB, protein disulphide-isomerase; MED24, mediator complex subunit 24; MED8, mediator complex subunit 8.

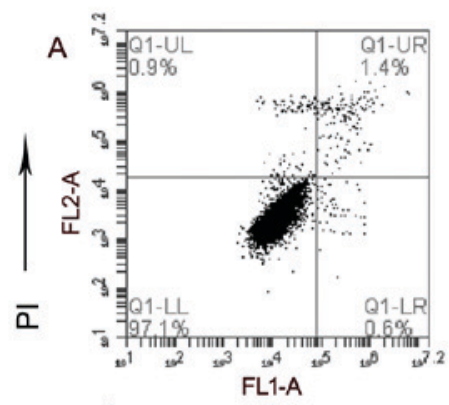

Annexin V-FITC

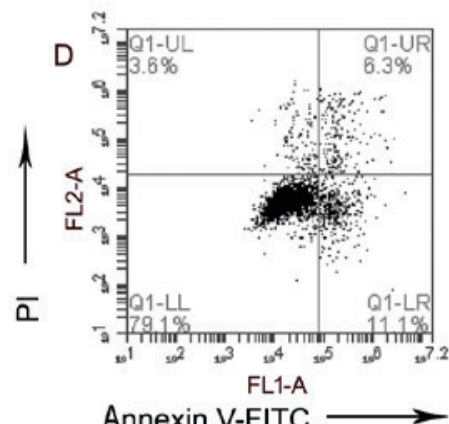

Annexin V-FITC

G

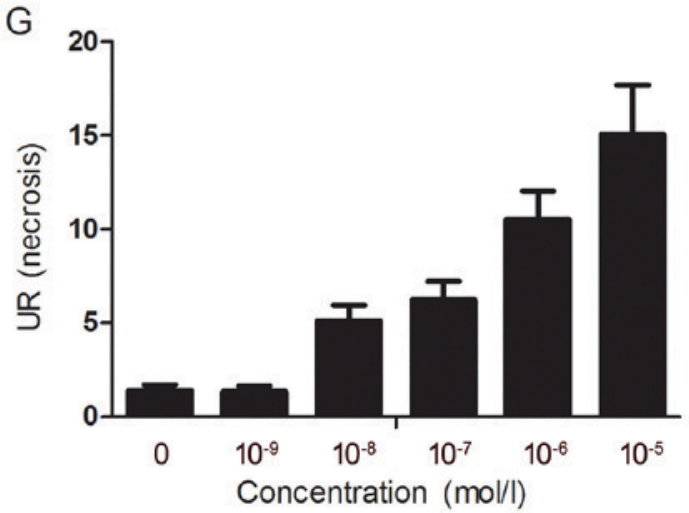

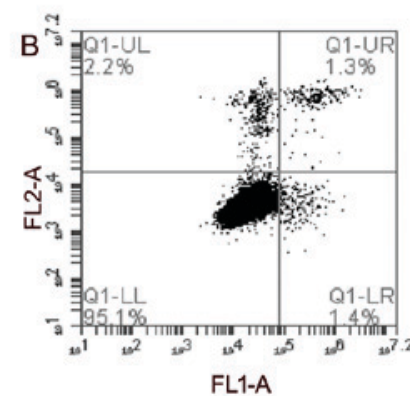
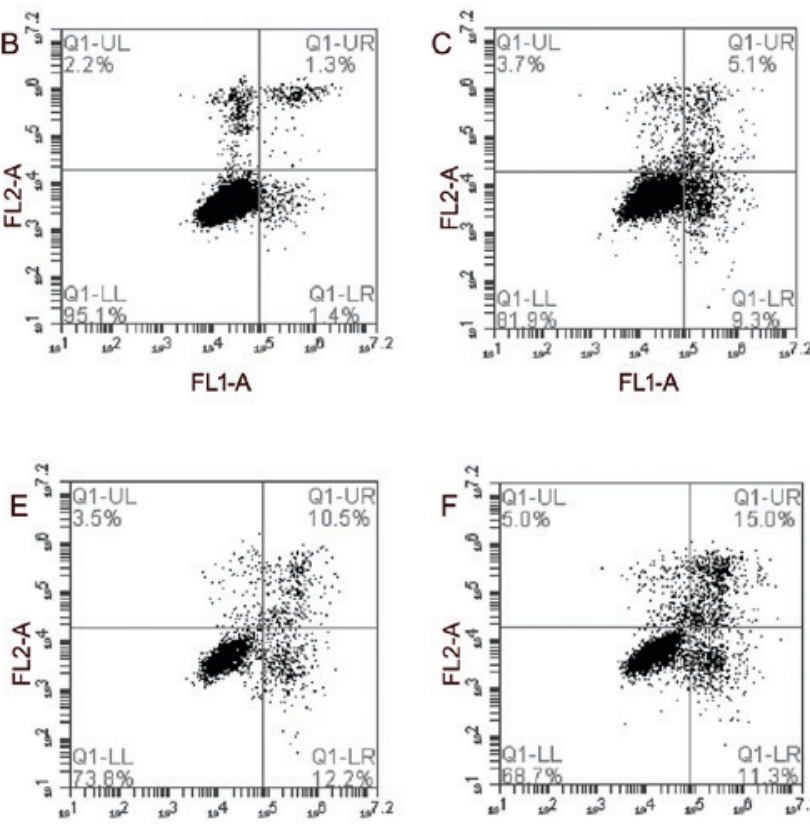

FL1-A

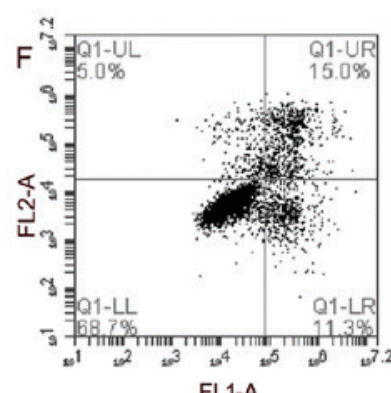

FL1-A

$\mathrm{H}$

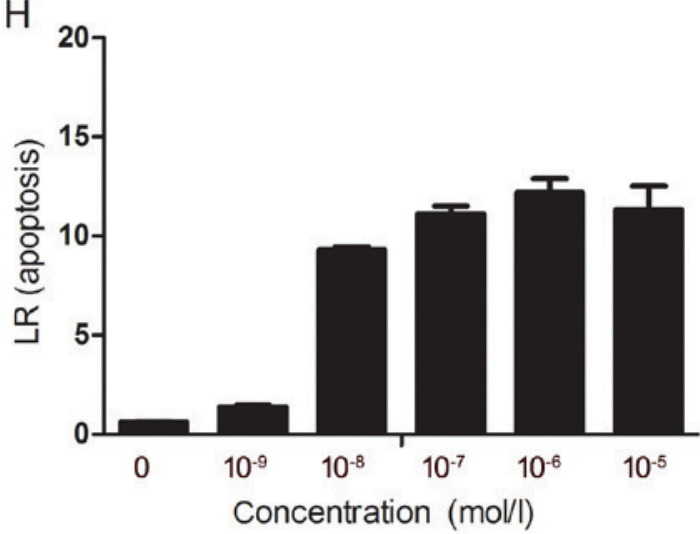

Figure 3. Effects of various concentrations of caerulein on the type of cell death observed in AR42J cells. Cells were treated with (A) 0 , (B) $10^{-9}$, (C) $10^{-8}$, (D) $10^{-7}$, (E) $10^{-6}$ or $(\mathrm{F}) 10^{-5} \mathrm{~mol} / \mathrm{l}$ caerulein to induce different degrees of acute pancreatitis. (G) Quantification of the percentage of necrotic cells in each group. UR, upper right quadrant. (H) Quantification of the percentage of apoptotic cells in each group. LR, lower left quadrant. The data are presented as the mean \pm standard deviation. 


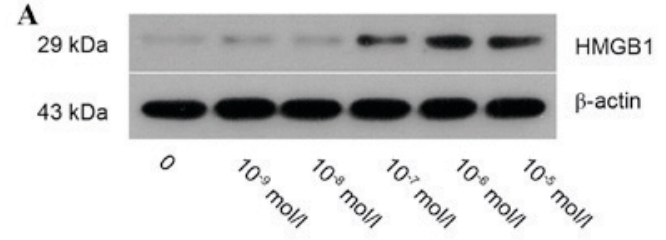

B

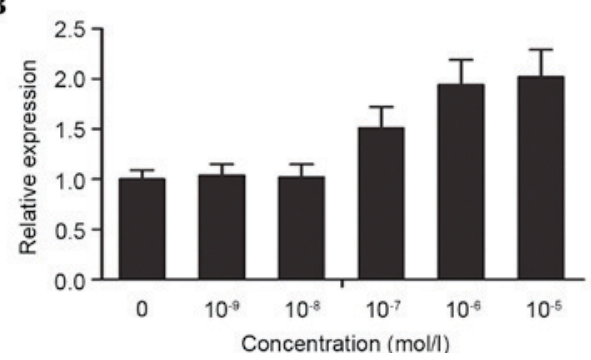

Figure 4. Effects of various concentrations of caerulein on HMBG1 protein expression levels in AR42J cells. (A) Representative western blot images and (B) quantification of HMGB1 protein expression levels in cells stimulated with various concentrations of caerulein. $\beta$-actin served as an interna control. The data are presented as the mean \pm standard deviation. HMGB1, high mobility group box-1 protein.

percentage of apoptotic cells as the concentration of caerulein increased from $10^{-8}$ to $10^{-5} \mathrm{~mol} / \mathrm{l}$ (Fig. $3 \mathrm{H}$ ).

Detection of HMGB1 in AR42J cells stimulated with various concentrations of caerulein. HGMB1 protein expression levels were detected by western blot analysis (Fig. 4A). The optical density value for the control group was set to 1 , and the values of HMGB1 and $\beta$-actin were calculated to obtain the relative expression levels in each group. HMGB1 protein expression levels increased in a dose-dependent manner. At $10^{-5} \mathrm{~mol} / \mathrm{l}$ caerulein, protein expression levels of HMGB1 were $>2$-fold those of the control group (Fig. 4B).

\section{Discussion}

AP is a common acute abdominal syndrome, but SAP is a disease with high morbidity and mortality. A variety of experimental studies and clinical trials have been performed to investigate the pathological process of AP. Notably, a previous study demonstrated that the type of pancreatic cell death may influence progression of the disease (9).

To examine the differences in protein expression associated with different types of cell death in AP, flow cytometry was performed following Annexin V/PI double staining to identify and sort apoptotic and necrotic cells for proteomics analysis. The results revealed that there were 28 different proteins specifically expressed in necrotic cells (VP group), including H2AFJ, DCP2, SGIP1, PMFBP1, YTHDF3, GM4944, PFDN2, HMGB1, TDRD5 and HCLS1. There were 14 different proteins specifically expressed in apoptotic cells (V group), including ERLEC1, RELT, ANXA2, P4HB, MCF2 and SLC39A8. Functional enrichment analysis was performed using DAVID to investigate which biological processes were associated with these proteins. In the bioinformatics analysis of apoptotic and necrotic cells, HMGB1 in the necrosis group was demonstrated to be involved in numerous biological processes, including formation of the chromosomal protein glycyl lysine isopeptide cross-link, and the positive regulation of phosphorylation, protein acid phosphorylation, the phosphate metabolic process and the phosphorus metabolic process. Therefore, it was hypothesized that HMGB1 serves important roles in necrosis to promote the occurrence and development of pancreatitis.

HMGB1 is a non-histone chromosome binding protein in eukaryotic cells. It is released from cells during necrosis and injury to interact with the membrane receptors receptor for advanced glycation end products, Toll-like receptor (TLR)2 and TLR4 (10-13). Previously, the function of HMGB1 in the developmental process of inflammation has received increasing attention $(14,15)$. Studies have indicated that extracellular HMGB1 is a novel pro-inflammatory cytokine in humans (16-18). Previous studies have confirmed that there is a clear association between extracellular HMGB1 and AP $(19,20)$. Yasuda et al (19) first reported that the serum level of HMGB1 in AP patients significantly increased in the initial $72 \mathrm{~h}$ of disease onset, and was associated with severity, infection and organ dysfunction of AP. In addition, serum HMGB1 levels demonstrated a positive correlation with serum lactate dehydrogenase, $\mathrm{C}$-reactive protein and total bilirubin. Therefore, HMGB1 may be used to estimate the prognosis of SAP patients, with high serum levels of HMGB1 being an indicator of poor prognosis.

To confirm the above results, the present study stimulated ARJ42 pancreatic cells with $10^{-9}, 10^{-8}, 10^{-7}, 10^{-6}$ or $10^{-5} \mathrm{~mol} / \mathrm{l}$ caerulein. The type of cell death was detected by flow cytometry. The results indicated that with increasing concentrations of caerulein, the percentage of necrotic cells gradually increased from 1.3 to $15.04 \%$. As the concentration of caerulein increased from $10^{-8}$ to $10^{-5} \mathrm{~mol} / \mathrm{l}$, the percentage of apoptotic cells rose from 9.3 to $12.1 \%$. A previous study demonstrated that apoptotic cells do not release HMGB1 (21) and do not induce an inflammatory reaction, as HMGB1 in apoptotic cells is not acetylated; therefore, HMGB1 is tightly bound to chromatin and will not be released into the extracellular matrix. By contrast, necrotic cells may passively release HMGB1 (21). HMGB1 loosely binds to the cell nucleus at the interphase and division phases of cell division. When cell necrosis occurs, cell membrane permeability increases and cell membrane integrity declines; therefore, HMGB1 rapidly leaks from the cells and is easily detected. To assess this, western blot analyses were performed in the present study to detect protein expression levels of HMGB1 in cells. The results demonstrated that with increased concentrations of caerulein, HMGB1 protein expression gradually increased, which was consistent with an increase in necrotic cells. Therefore, at the early stage of pancreatitis, HMGB1 may be involved in necrosis as a pro-inflammatory cytokine.

The present study confirmed that there was an association between HMGB1 and necrotic cells of AP. Yu et al (22) used classic retrograde pancreatic duct injection of $5 \%$ sodium taurocholate to induce a rat AP model. Animals were sacrificed after 3, 6, 12 or $24 \mathrm{~h}$, and peripheral blood samples and pancreatic tissue samples were collected to detect the levels of inflammatory factors. The results demonstrated that early-stage inflammatory factors including interleukin (IL)-1, tumour necrosis factor (TNF)- $\alpha$ and IL- 6 increased rapidly, whereas the production, peak and regression time of HMGB1 was slightly delayed. Therefore, HMGB1 may be involved in 
the early-stage inflammatory reaction and serve important roles in the prolongation and maintenance of inflammatory reactions $(22,23)$. In the present study, a significant increase in HMGB1 levels were detected after $8 \mathrm{~h}$ of stimulation of pancreatic acinar cells with caerulein; this result supported the hypothesis that HMGB1 is involved in early-stage inflammatory reactions. Considering the number of apoptotic cells, the $8 \mathrm{~h}$ timepoint was selected (8). However, spatial and temporal differences of HMGB1 expression also exist in AP (22), detection at multiple timepoints would aid the understanding of other differentially expressed proteins. Future studies are required to elucidate this. Yasuda et al (24) proposed the 'HMGB1 circulation' hypothesis. At the early stage of SAP, HMGB1 is primarily produced by pancreatic and peritoneal macrophages. HMGB1 is subsequently released into the blood and causes distant organ damage. The injured organs may additionally release HMGB1, producing a cyclic reaction. Therefore, HMGB1 inhibitors may have therapeutic effects on AP (20). The inhibitors of HMGB1, ethyl pyruvate and pyrrolidine dithiocarbamate, may inhibit nuclear factor- $\kappa \mathrm{B}$ activation (25), thus reducing HMGB1 levels and blocking HMGB1 circulation; therefore, these inhibitors may protect against the dysfunction of a variety of organs (26-30).

In conclusion, the present study used a pancreatic acinar cell AP model to investigate the expression of HMGB1. The results of the proteomics analysis revealed that HMGB1 protein expression levels differed significantly between apoptotic and necrotic cells. Therefore, as a pro-inflammatory cytokine, HMGB1 may be involved in inflammation and promote cell death by necrosis. The biological processes involving HMGB1 were identified, and provide an experimental basis for clinical intervention in AP. However, the association between HMGB1 and other early-stage inflammatory mediators, cellular processes and specific pathogenic underlying mechanisms remain to be fully elucidated, and require further study.

\section{Acknowledgements}

The present study was supported by a grant from the National Natural Science Foundation of China (grant no. 81370566).

\section{References}

1. Petersen OH, Tepikin AV, Gerasimenko JV, Gerasimenko OV, Sutton R and Criddle DN: Fatty acids, alcohol and fatty acid ethylesters: Toxic $\mathrm{Ca} 2+$ signal generation and pancreatitis. Cell Calcium 45: 634-642, 2009.

2. Keck T, Friebe V, Warshaw AL, Antoniu BA, Waneck G, Benz S, Hopt UT and Fernández-del-Castillo C: Pancreatic proteases in serum Leukocyte-endothelial adhesion and pancreatic microcirculatory failure. Pancreatology 5: 241-250, 2005.

3. Leung PS and Chan YC: Role of oxidative stress in pancreatic inflammation. Antioxid Redox Signal 11: 135-165, 2009.

4. Golstein P and Kroemer G: Cell death by necrosis: Towards a molecular definition. Trends Biochem Sci 32: 37-43, 2007.

5. Rinderknecht H: Fatal pancreatitis, a consequence of excessive leukocyte stimulation? Int J Pancreatol 3: 105-112, 1988.

6. Ziangirova GG and Antonova OV: The causes of necrobiosis and apoptosis of corneal epithelial cells during primary acquired keratoconus. Izv Akad Nauk Ser Biol 5: 517-521, 2002 (In Russian).

7. Andersson R and Wang XD: Patterns of pancreatic cell death: Apoptosis versus oncosis. Pancreas 17: 281-288, 1998.

8. Chu J, Ji H, Lu M, Li Z, Qiao X, Sun B, Zhang W and Xue D: Proteomic analysis of apoptotic and oncotic pancreatic acinar AR42J cells treated with caerulein. Mol Cell Biochem 382: 1-17, 2013.
9. Bhatia M: Apoptosis versus necrosis in acute pancreatitis. Am J Physiol Gastrointest Liver Physiol 286: G189-G196, 2004.

10. Hori O, Brett J, Slattery T, Cao R, Zhang J, Chen JX, Nagashima M, Lundh ER, Vijay S, Nitecki D, et al: The receptor for advanced glycation end products (RAGE) is a cellular binding site for amphoterin. Mediation of neurite outgrowth and co-expression of rage and amphoterin in the developing nervous system. J Biol Chem 270: 25752-25761, 1995.

11. Park JS, Svetkauskaite D, He Q, Kim JY, Strassheim D, Ishizaka A and Abraham E: Involvement of toll-like receptors 2 and 4 in cellular activation by high mobility group box 1 protein. J Biol Chem 279: 7370-7307, 2004.

12. Urbonaviciute V, Fürnrohr BG, Meister S, Munoz L, Heyder P, De Marchis F, Bianchi ME, Kirschning C, Wagner $\mathrm{H}$, Manfredi AA, et al: Induction of inflammatory and immune responses by HMGB1-nucleosome complexes: Implications for the pathogenesis of SLE. J Exp Med 205: 3007-3018, 2008.

13. Park JS, Gamboni-Robertson F, He Q, Svetkauskaite D, Kim JY, Strassheim D, Sohn JW, Yamada S, Maruyama I, Banerjee A, et al: High mobility group box 1 protein interacts with multiple Toll-like receptors. Am J Physiol Cell Physiol 290: C917-C924, 2006.

14. Goodwin GH, Sanders C and Johns EW: A new group of chromatinassociated proteins with a high content of acidic and basic amino acids. Eur J Biochem 38: 14-19, 1973.

15. Wang H, Bloom O, Zhang M, Vishnubhakat JM, Ombrellino M, Che J, Frazier A, Yang H, Ivanova S, Borovikova L, et al: HMG-1 as a late mediator of endotoxin lethality in mice. Science 285: 248-251, 1999.

16. Wang H, Yang H, Czura CJ, Sama AE and Tracey KJ: HMGB1 as a late mediator of lethal systemic inflammation. Am J Respir Crit Care Med 164: 1768-1773, 2001.

17. Magna M and Pisetsky DS: The role of HMGB1 in the pathogenesis of inflammatory and autoimmune diseases. Mol Med 20: 138-146, 2014.

18. Singh A, Feng Y, Mahato N, Li J, Wu C and Gong J: Role of high-mobility group box 1 in patients with acute obstructive suppurative cholangitis-induced sepsis. J Inflamm Res 8: 71-77, 2015.

19. Yasuda T, Ueda T, Takeyama Y, Shinzeki M, Sawa H, Nakajima T, Ajiki T, Fujino Y, Suzuki Y and Kuroda Y: Significant increase of serum high-mobility group box chromosomal protein 1 levels in patients with severe acute pancreatitis. Pancreas 33: 359-363, 2006.

20. Sawa H, Ueda T, Takeyama Y, Yasuda T, Shinzeki M, Nakajima T and Kuroda Y: Blockade of high mobility group box-1 protein attenuates experimental severe acute pancreatitis. World J Gastroenterol 12: 7666-7670, 2006.

21. Scaffidi P, Misteli T and Bianchi ME: Release of chromatin protein HMGB1 by necrotic cells triggers inflammation. Nature 418: 191-195, 2002.

22. Yu C, Huang L, Li X, Zhu H, Li Z and Yu X: Spatial and temporal differences of HMGB1 expression in the pancreas of rats with acute pancreatitis. Int J Clin Exp Pathol 8: 6928-6935, 2015.

23. Biscetti F, Ghirlanda G and Flex A: Therapeutic potential of high mobility group box-1 in ischemic injury and tissue regeneration. Curr Vasc Pharmacol 9: 677-681, 2011.

24. Yasuda T, Ueda T, Shinzeki M, Sawa H, Nakajima T, Takeyama Y and Kuroda Y: Increase of high-mobility group box chromosomal protein 1 in blood and injured organs in experimental severe acute pancreatitis. Pancreas 34: 487-488, 2007.

25. Sah RP and Saluja A: Molecular mechanisms of pancreatic injury. Curr Opin Gastroenterol 27: 444-451, 2011.

26. Bünger R, Mallet RT and Hartman DA: Pyruvate-enhanced phosphorylation potential and inotropism in normoxic and postischemic isolated working heart. Near-complete prevention of reperfusion contractile failure. Eur J Biochem 180: 221-233, 1989.

27. Cicalese L, Lee K, Schraut W, Watkins S, Borle A and Stanko R: Pyruvate prevents ischemia-reperfusion mucosal injury of rat small intestine. Am J Surg 171: 97-101, 1996.

28. Sileri P, Schena S, Morini S, Rastellini C, Pham S, Benedetti E and Cicalese L: Pyruvate inhibits hepatic ischemia-reperfusion injury in rats. Transplantation 72: 27-30, 2001.

29. Miyaji T, Hu X, Yuen PS, Muramatsu Y, Iyer S, Hewitt SM and Star RA: Ethyl pyruvate decreases sepsis-induced acute renal failure and multiple organ damage in aged mice. Kidney Int 64: 1620-1631, 2003

30. Satoh A, Shimosegawa T, Fujita M, Kimura K, Masamune A, Koizumi $\mathrm{M}$ and Toyota T: Inhibition of nuclear factor-kappaB activation improves the survival of rats with taurocholate pancreatitis. Gut 44: 253-258, 1999. 